\title{
A MONTE CARLO SIMULATION STUDY ON THE DOME EFFECT
}

\author{
R. Roncella ${ }^{1 *}$, G. Forlani ${ }^{1}$, F. Diotri ${ }^{1}$ \\ ${ }^{1}$ Department of Engineering and Architecture, Campus Area delle Scienze 181/A, 20145 Parma, Italy - (riccardo.roncella, \\ gianfranco.forlani)@unipr.it, fabrizio.diotri@gmail.com
}

Commission TCII, WG II/1

KEY WORDS: UAV photogrammetry, Dome effect, Bowl effect, Monte Carlo simulation, Block control.

\begin{abstract}
:
A dome-shape deformation has been found to affect the photogrammetric surface reconstruction in several real and simulated experiments. Its origin has been recognised in inaccurate estimation of the camera parameters and many papers already concentrated on conditions to avoid its development, especially as far as block design is concerned. This paper presents a Monte Carlo simulation to investigate surface reconstruction elevation errors in UAV (Unmanned Aerial Vehicle) photogrammetric blocks. The simulation tests are designed to find out the effect of block shape, camera axis inclination, side-lap, cross strips addition and block control by GCP or GNSS-assisted on the extent of the deformations. The main findings are: i) that GNSS-assisted blocks are generally more robust compared to GCP-controlled ones; ii) that, in GNSS-assisted blocks, unless a mix of nadiral and inclined strips is present, at least one fixed GCP must be provided; iii) that cross strip can conveniently be slimmed to save flight time and processing time; iv) that the effectiveness of GNSS deteriorate as the block shape slims out.
\end{abstract}

\section{INTRODUCTION}

One of the primary goals of UAV surveys being the production of high resolution DTM (Digital Terrain Models), a major concern is how to avoid systematic errors in the point cloud obtained from dense matching after block orientation with Structure from Motion (SfM) algorithms.

In a simulation experiment in close range photogrammetry (Wackrow and Chandler, 2008), found "residual systematic error surfaces or "domes", discernible in DEM (Digital Elevation Models), caused by slightly inaccurate estimated lens distortion parameters". Moreover, such error surfaces could be minimised using a mildly convergent camera configuration.

Reducing or avoiding the "dome" (or "bowl") effect has since been a topic in UAV photogrammetry as evidence of systematic errors arose from many tests. To avoid projective coupling with E.O. (Exterior Orientation) parameters, especially with nadiral imaging, pre-calibration would be advisable. Pre-calibration is well suited when the camera is mechanically stable and repeatable in focusing operations (Cramer et al., 2017); a further constraint is that it should be operated in the field under similar conditions (image scale, scene depth, etc.) to that of calibration. As UAV typically host consumer cameras, most users prefer onthe-job calibration through a self-calibrating Bundle Block Adjustment (BBA), as I.O. (Interior Orientation) parameters stability in such cameras is uncertain.

In these contexts one of the strategy proposed in (Girod and Filhol, 2020) of using an extended camera model considering the work by (Tournadre et al., 2015), can be counter-productive, especially with critical configurations (e.g., without cross strips or oblique images), as observed by the cited authors themselves, leading, most likely, to an over-parametrization of the BBA and increasing the occurance of projective coupling with I.O. and E.O. parameters.
Conditions for successful on-the-job calibration in UAV projects are discussed in many papers (Harwin 2015, James 2017, Cramer 2017, Fraser, 2018). In particular, convergent (oblique) images or imaging with a mild inclination of camera axis in forward direction have been shown to be effective (James and Robson 2014; Nesbit and Hugenholtz, 2019; Taddia et al., 2020, SanzAblanedo et al., 2020, Radford and Bevan, 2020).

Though GCP are not needed for camera calibration (Carbonneau and Dietrich, 2017), in fact the on-the-job-calibration outcome (and so does the "dome effect") may be influenced by GCP density and distribution (Sanz-Ablanedo et al., 2018).

In GNSS-assisted block orientation (Ackermann, 1993; Bilker et al., 1998), camera parameters update is even more critical, as residual calibration errors are likely to affect primarily ground point coordinates (Cramer et al., 2000; Daakir et al., 2015, Forlani et al., 2020).

A study, based on Monte Carlo simulations, by (James et al., 2017) aiming to produce precision maps of a real block under varying ground control strength and type (with GCP or by GNSS (Global Navigation Satellite System) on-board) found surface shape errors (doming) despite using GCP in the self-calibrating BBA. This study has a similar goal, though with a partially different set of parameters characterising the UAV blocks. After (Taddia et al., 2020) experiment, it was also found by others (Stott et al., 2020) that acquiring longitudinal strips with the camera axis inclined in forward direction, rather than strictly nadiral, is beneficial, especially in case of GNSS-assisted block orientation. One of this paper goals is therefore to look at pro and cons of such choice. Moreover, rather than concentrating on a single block shape, we wanted to explore a (albeit small) range of block shapes, as in particular we were interested in the effectiveness of using GNSS-assisted orientation in cases close to corridor mapping, following a previous test (Forlani et al., 2019).

\footnotetext{
* Corresponding author
} 
In particular, the following project parameters/configurations are considered:

- $\quad$ rectangular block shape, with increasing width-to-

height ratios.

camera axis inclination: nadiral, inclined.

amount of forward and side overlap.

addition of cross strips.

GCP or GNSS-assisted block control.

GCP placing and spacing.

The goal is to evaluate the relative importance of each parameter and possibly find out the most efficient combination of overlap, camera imaging geometry and block control that minimizes the "dome effect".

\section{MATERIALS AND METHODS}

\subsection{Test organization}

The simulation tests are organised as follows. Four basic strip modules are first defined (see also Table 1):

- LN: nine nadiral longitudinal strips.

- LI: nine longitudinal strips with off-nadir camera axis inclination of $15^{\circ}$ in forward direction.

- CN: seven nadiral cross strips with 50\%-50\% overlap.

- CI: seven cross strips with off-nadir camera axis inclination of $15^{\circ}$ in forward direction and $50 \%-50 \%$ overlap.

LN and LI come in two versions: with $80 \%-80 \%$ overlap (indicated in the following as LN_8 or LI_8) and with $80 \%-60 \%$ overlap (LN_6 or LI_6).

\begin{tabular}{|c|l|l|l|}
\hline $\begin{array}{c}\text { Block } \\
\text { module }\end{array}$ & $\begin{array}{c}\text { Strip } \\
\text { type }\end{array}$ & $\begin{array}{c}\text { camera off-nadir } \\
\text { inclination }\end{array}$ & $\begin{array}{c}\text { Overlap \% } \\
\text { forward-side }\end{array}$ \\
\hline LN & Longit. & Nadiral & $\begin{array}{l}80-80 \text { (LN_8) } \\
80-60 \text { (LN_6) }\end{array}$ \\
\hline LI & Longit. & $\begin{array}{l}15^{\circ} \text { forward } \\
\text { direction }\end{array}$ & $\begin{array}{l}80-80 \text { (LI_8) } \\
80-60 \text { (LI_6) }\end{array}$ \\
\hline CN & Cross & Nadiral & $50-50$ \\
\hline CI & Cross & $\begin{array}{l}15^{\circ} \text { forward } \\
\text { direction }\end{array}$ & $50-50$ \\
\hline
\end{tabular}

Table 1 - Basic block modules for the simulations

Six block configurations will be tested, made of the following strip modules combinations: LN, LI, LN-CN, LI-CI, LN-CI, LI$\mathrm{CN}$. Each block module is tested with both overlap configurations for LN and LI.

Blocks with three different surveyed areas, changing width-toheight ratios (BWH) of the area itself, are obtained, discarding from the original block, some longitudinal strips. First four and then two more longitudinal strips are discarded from the original $80 \%$ side-lap blocks: in such a way blocks of five and of three strips are generated (Table 2).

\begin{tabular}{|c|c|}
\hline Denomination & $\begin{array}{c}\text { \# strips } \\
80 \%-60 \%\end{array}$ \\
\hline BWH_1 & $9-5$ \\
\hline BWH_2 & $5-3$ \\
\hline BWH_3 & $3-2$ \\
\hline
\end{tabular}

Table 2 - Three different rectangular block shapes considered, made from 9 strips to 3 strips with $80 \%$ side-lap (and the respective $60 \%$ side-lap)

The number of cross strips remains unchanged for all different width-to-height ratios, while their lengths are cut accordingly. Combining configurations (6 different blocks) with overlap percentages ( 2 different overlaps) and width-to-height ratios (3 areas with different size) a total of 36 cases is produced.

Two block control strategies are examined each one with two different configurations: ground (GCP) and aerial (GNSS). In the first GCP configuration 4 GCP are placed at the block corners; in the other an additional GCP located at the block centre is considered. In the GNSS case, all camera stations are used as control; in the second control configuration a single GCP, located at the block centre, is fixed. Overall $36 \times 4=144$ cases are examined.

\subsection{Real data acquisition}

The synthetic datasets of the simulation are built from suitably exploiting the BBA output of a real block, flown according to the above-described LN, LI, CN, CI modules. The goal is to avoid ending with an unrealistic distribution of the tie points over a regular grid and with an artificially high and too homogeneous distribution of ray multiplicity (i.e., the number of images a point is observed on).

A gently undulated area (Figure 1) of about $400 \mathrm{~m}$ by $180 \mathrm{~m}$, mainly covered in grass has been surveyed with a Dji Phantom IV Pro equipped with a FC6310 camera with an $8.8 \mathrm{~mm}$ lens (focal length $24 \mathrm{~mm}$ as $35 \mathrm{~mm}$ format equivalent) and a resolution of $5472 \times 3648$ pixel (pixel size 2.41 micrometres).

Four different image blocks were acquired on the same day in a very short period of time (ca. one hour). The first flight plan is composed of nine longitudinal strips, each composed by 32 images and was repeated with nadir (LN) and off-nadir (LI) camera axis inclination. The second series (nadir $(\mathrm{CN})$ and offnadir (CI)) acquires seven cross strips, each composed by 7 images. Both longitudinal and cross strips are flown at an altitude above ground level of about $65 \mathrm{~m}(\mathrm{GSD}=1.8 \mathrm{~cm})$.

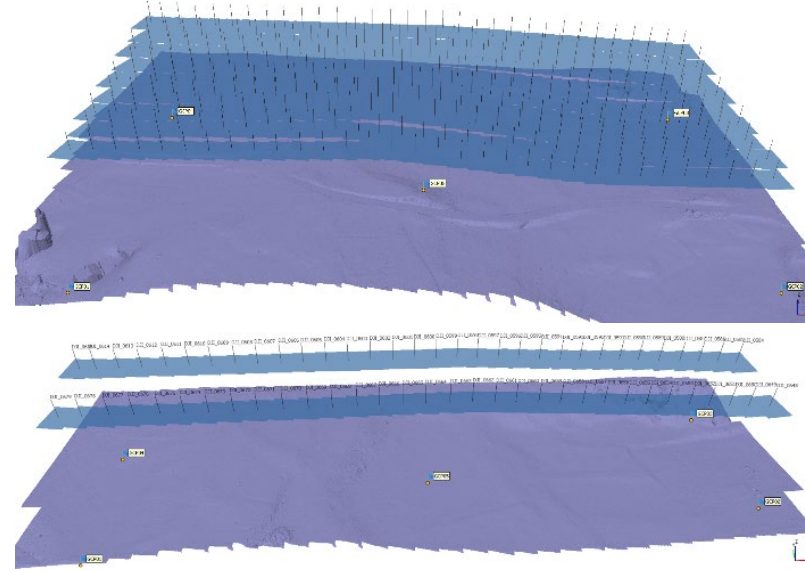

Figure 1. Top: view of the BWH 1 LN 8 configuration (nine strips, $80 \%$ side-lap) with $5 \mathrm{GCP}$; bottom: view of the BWH_3

LN 6 configuration (two strips, $60 \%$ side-lap) with 5 GCP

Combining the images of the four different photogrammetric blocks, the six configurations illustrated in the previous sections are obtained. Next, the six image blocks are oriented in Agisoft Metashape (Agisoft, 2021) v. 1.7.1 and the tie-points extracted along with their corresponding image points are exported. In this way, in the simulations, the actual distribution of tie points can mimic the real-world image block. All 36 combinations of configurations (6), overlaps (2 combinations - removing one every two strips to obtain the $60 \%$ side-lap blocks) and width-toheight ratios ( 3 combinations - removing the first half of $2 / 3$ of the original image block) have been formed. 


\subsection{Synthetic datasets generation}

The estimated values of the I.O., E.O. and distortion parameters and of the tie points ground coordinates are taken from the real block output and are treated as true values for the subsequent simulations. Having exported, for each of the six different block modules, the list of tie points their corresponding image coordinates true values have been generated by projecting the ground point coordinates with the collinearity equations, according to the estimated image exterior orientation parameters and to the camera parameters. The synthetic image coordinates so obtained incorporate the optical and sensor frame distortion estimated for the real block.

Normally distributed errors with standard deviations reported in Table 3 have been generated in each run of the MC simulation and added to the observations.

\begin{tabular}{|c|c|c|}
\hline $\begin{array}{c}\text { Camera Station } \\
(\mathrm{cm})\end{array}$ & GCP $(\mathrm{cm})$ & $\begin{array}{c}\text { Tie point image } \\
(\text { pix })\end{array}$ \\
\hline 3.0 & 0.5 & 1 \\
\hline
\end{tabular}

Table 3 - Standard deviations of the normally distributed errors added to the observations.

In principle the standard deviations of camera stations and GCP coordinates assigned to the observations in the BBA should match the values in Table 2. For the GCP and the camera station coordinates observations, the values have been reduced by a factor three, as it has already been noticed by several authors (Benassi et al., 2016, Fraser, 2018) that the much larger number of image observations with respect to other observations needs to be counterbalanced by increasing the weights of the latter. In each simulation run, the appropriate block control (4 or 5 GCP for the ground control case; all camera station and one or no GCP) is applied. Then, a self-calibrating BBA of the block configuration is executed, and its results stored. As the procedure is repeated 1000 times for each of the 144 block configurations. Consequently, 144000 different image block orientations have been computed.

\subsection{Simulation results evaluation}

As the paper focus is on block deformations in elevation, the results are summarized on tie points ground coordinates and, to highlight the "dome effect", on a regular grid of check points distributed over the area. In both cases, the estimated coordinates are compared to the true ones. The reason for introducing this "double check" is argued in the following. Though in principle the errors measured at tie points are true errors, their amount depends, among other factors relevant to "dome effect" (e.g., distance from the nearest GCP), on image matching accuracy, terrain shape, redundancy of observations (i.e., number of images observing the point, intersection angles between rays, tie point density in the vicinity, etc.). To smooth out these effects with respect to the contribution of orientation errors (i.e., the main cause of the dome effect), the image coordinates of the check points are not corrupted (no random observation error is added) and are not included in the BBA. Their estimated ground coordinates are in fact obtained by forward intersection from three homologous rays that ensure an average quality of the intersection geometry over the whole check points grid.

For each run of the simulation, and for each of the 144 configurations, the individual errors on tie and check points are available as well as general statistics per configuration: average error, error standard deviation, max and min error, RMSE and error histograms for each coordinate.

To summarize the results, two main statistics will be reported: the average Root Mean Square Error (RMSE) and the average maximum $\mathrm{Z}$ error (maxZE) on check points, both expressed in GSD units (for all the tests the GSD is equal to $1.8 \mathrm{~cm}$ ). The mean RMSE is computed as follows: for each single run, the RMSE of the differences between the estimated check point coordinates and their corresponding true values is computed. The mean RMSE, reported in the subsequent sections, is the average value of the 1000 RMSE computed for each test combination. The RMSE within a simulation run measures the average error over all the check points; its average over the 1000 runs, therefore, can be thought of as the expected average elevation error over the area.

Likewise, the mean maximum $\mathrm{Z}$ error is computed as such: for each of the 1000 runs of the MC simulations, the maximum (absolute) error on the check points is computed and averaged over the 1000 runs of the MC simulations. The maximum error registered in each simulation run measures the maximum amount of deformation in elevation; as an extreme value, rather than the maximum over all simulations, its average over the 1000 runs has been considered representative of the expected largest elevation error over the area. It should be pointed out, however, that maxZE is not a measure of the "dome height", as it is not referred to a specific point located in the area nor is an interpolated value of an error surface fitting the error surface.

Moreover, in two of the control cases foreseen in the test (see 2.1) one GCP is placed in the block centre for both the GCP (5 GCP) and the GNSS (1 GCP) cases. This is done exactly to find out the "dampening" effect that this might produce on the dome effect: therefore, comparison between the results of these two control cases should keep this in mind.

One of the paper goals being also to find out whether, all else equal, slimming the block from nine to five strips (three with $60 \%$ side-lap) and then to three strips (two with $60 \%$ side-lap), while keeping the longitudinal strip length unchanged, affects the sensitivity to systematic errors in elevation. To assess whether the slimmer block undergoes to larger deformations in elevations, the RMSE of corresponding block configurations will be compared.

\section{RESULTS}

All the simulations results are recorded in a PostgreSQL database for easily querying the data. In the following, we divided the 144000 error sets in 144 groups, each corresponding to a different simulation (i.e., combination of block module (6), control strategy (2), number of GCP (2), side-lap (2) and surveyed area width-to-height ratio BWH (3)).

For sake of simplicity, results are presented in three separate sections, the first referring to the full block configuration (BWH 1 ratio). The second and the third summarize, through a comparison between respectively BWH_1 and BWH_2, and between BWH_1 and BWH_3, the influence on "dome effect" of considering a "thinner" block. It should be noted that in blocks BWH_2 and BWH_3, the length of the image block along the longitudinal strip direction, as well as the distances between the GCP along the same direction, is always the same. As pointed out in the previous section, the aim in these simulations is to evaluate if thinning the number of strips and shortening consequently the cross strips (if considered), therefore reducing the actual rigidity of the block, increases the insurgence of the "dome effect".

\subsection{Block BWH_1}

Figure 2 shows the RMSZ (RMS of the $\mathrm{Z}$ coordinate), expressed in GSD units, for the six configurations with different side-lap and block control separately for the GCP (top) and GNSS (bottom) case, respectively. In all this paper plots, the blue bars represent the $80 \%$ case with 5 and 1 GCP respectively in the GCP 
and GNSS cases. The grey ones still the $80 \%$ case but with $4 \mathrm{GCP}$ only and none in the GCP and GNSS case, respectively. The red and orange bars are the corresponding cases with $60 \%$ side-lap.

GCP - RMSZ (GSD)

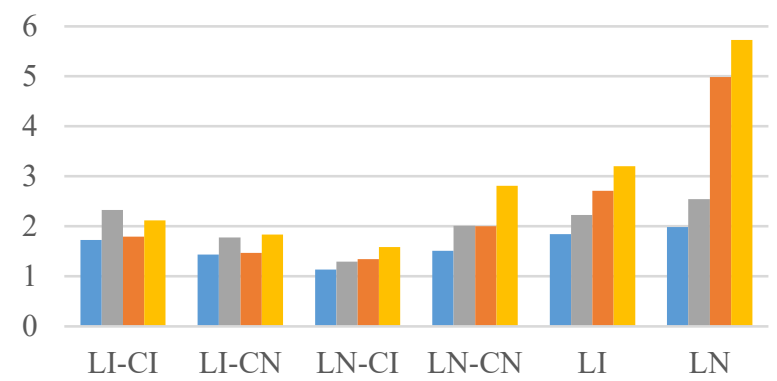

GNSS - RMSZ (GSD)

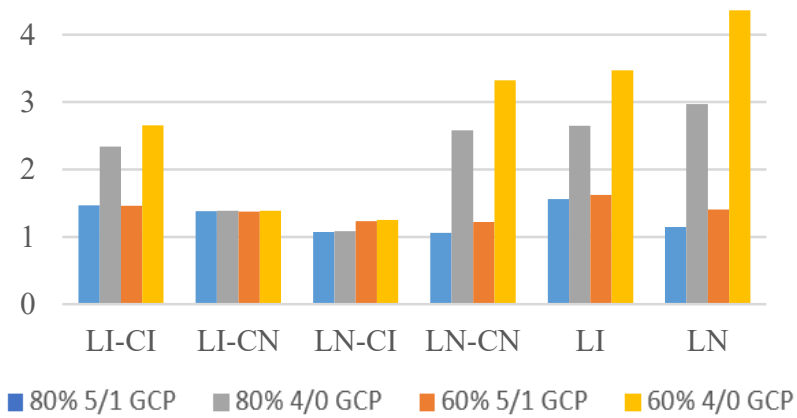

Figure 2. RMSZ in GSD units for the six basic configurations in the GCP (top) and GNSS (bottom) cases.

As far as the GCP-controlled image blocks are concerned (Figure 2 top), the configurations with cross and longitudinal strips achieve better performances than the other ones. As one can expect, the more rigid (i.e., $80 \%$ side-lap) and controlled (5 GCP) the block, the better the results obtainable. The increment in RMSZ removing the central GCP (using, in other words, only 4 GCP) is more evident for LI-CI and LN-CN (ca. 35\%), and for LN (ca. 28\%). Reducing the side-lap (to $60 \%$ ), the first two configurations seem not to be affected: quite surprisingly, for LICI with 4 GCP the results with $60 \%$ side-lap is actually $10 \%$ better. On the contrary LN-CN $(+36 \%)$, LI $(+46 \%)$ and especially $\mathrm{LN}(+140 \%)$ seems more dependent on the rigidity (in terms of image overlap) of the block.

Also, for GNSS-controlled image blocks, the configurations with cross and longitudinal strips achieve better performances, although the differences are smaller as long as 1 GCP at block centre is added. Reducing the side-lap, unlike the GCP case, does not affect significantly the RMSZ, especially if one GCP and cross strips are provided.

With $80 \%$ side-lap (blue and red bars) the accuracy is the best in both GCP and GNSS cases, with an average 2.0 GSD for the former and $1.3 \mathrm{GSD}$ for the latter. With $60 \%$ side-lap (grey and orange bars) the accuracy is on average 2.6 GSD for the GCP case and 2.1 GSD for the GNSS case. On the other hand, the accuracy in the GNSS case without GCP (grey bars), is markedly worse than with 1 GCP. Without this central GCP i.e., using exclusively the camera station position for ground control, only the mixed LI-CN and LN-CI combinations seem not being significantly affected, while for all the other configurations the RMSZ increment is quite strong: $+71 \%$ on average for LI-CI and up to $185 \%$ for $\mathrm{LN}$.
Removing the only single GCP in the GNSS case means that the image block control relies entirely on the camera station locations and on the quality of the I.O. parameters estimated during the onthe-job calibration. If, for instance, a wrong principal distance is estimated, a systematic depth error arises for all the estimated ground points (tie and check points). Since all the CP are located at the same distance along $\mathrm{Z}$ direction from the camera station, this systematic error affects the ground points increasing (or decreasing) uniformly their $\mathrm{Z}$ coordinate. Other I.O. wrongly estimated parameters may affect the results differently, but always producing systematic errors.

Removing the single GCP always entails an increased mean error on the CP coordinates. Figure 3 shows the Standard Deviation (SD) of the elevation error for the six configurations, considering the GNSS-assisted image block.

\section{GNSS - Z error SD (GSD)}

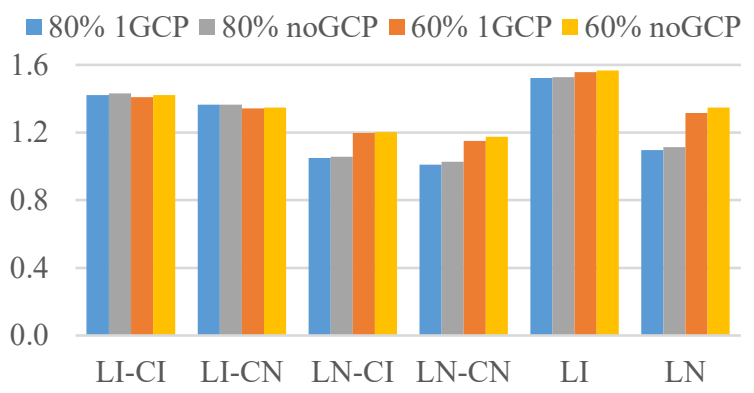

Figure 3. Average standard deviation of $Z$ differences in GSD units for the six configurations in the GNSS

Comparing the RMSE (Figure 2 bottom) with the corresponding standard deviation of the $\mathrm{Z}$ differences (Figure 3 ) it is evident that the strong differences, in particular for LI-CI, LN-CN, LI and $\mathrm{LN}$, shown by the RMSE when the single GCP is removed, do not correspond to similar differences in the standard deviations. This implies that the actual shape of the "dome" or "bowl" is not strongly affected by the removal of the single GCP. Rather, the entire object, due to the I.O. parameters estimation errors caused, most likely, by stronger correlations between the parameters, is shifted along $Z$ direction systematically. Figure 4 shows the average of $Z$ differences (the $Z$ error) as percentage of the RMSE in the GNSS case. When the single GCP is present, the systematic error component is small (max value: $11 \%$ in $\mathrm{LN}$ configuration). Without the GCP the systematic component becomes dominant: up to $90 \%$ in $\mathrm{LN}$ configuration. More importantly, not all combination of oblique and nadiral imaging effectively prevent large errors. Indeed, only LI-CI and LN-CI are unaffected, while LI-CI and LN-CN are both, though to a different degree.

GNSS - Mean error as \% of RMSZ
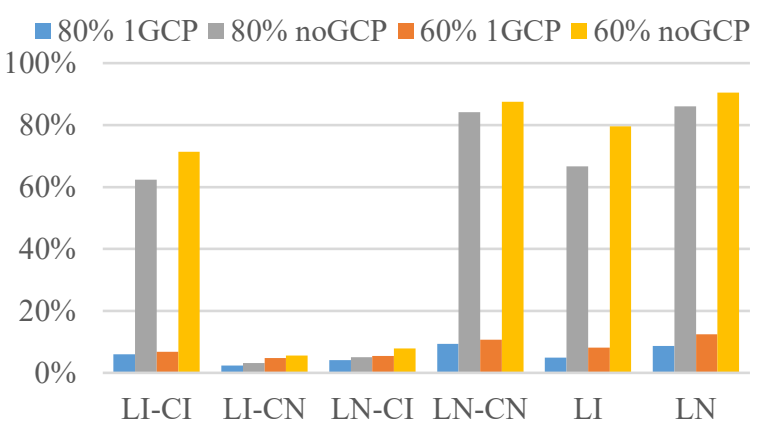

Figure 4. Mean of $Z$ differences as percentage of the RMSZ for the six configurations in the GNSS case. 
Combining nadiral and off-nadir longitudinal and cross strips (LI-CN or LN-CI) the systematic errors are almost negligible. On the contrary, the other configurations are affected by strong systematic errors, confirmed by the fact that, for these image block type the estimated I.O. parameters are much more variable. Table 4, for instance, reports the standard deviations of the focal length for the six configurations with and without the single GCP.

\begin{tabular}{|c|c|c|c|c|c|c|}
\cline { 2 - 7 } \multicolumn{1}{c|}{} & LI-CI & LI-CN & LN-CI & LN-CN & LI & LN \\
\hline 1 GCP & 0.38 & 0.22 & 0.28 & 0.48 & 0.39 & 0.50 \\
\hline 0 GCP & 2.10 & 0.23 & 0.29 & 3.65 & 2.46 & 4.34 \\
\hline
\end{tabular}

Table 4 - Standard deviations of the estimated focal length (in pixel) in the six configurations for GNSS-assisted blocks.

The results seem to confirm the behaviours noticed, for instance, by (Benassi et al, 2017) and (Hugenholtz, 2016). The problem has been addressed by some authors suggesting the use of oblique imaging. In particular, in (Taddia et al, 2020; Stott et al., 2020) forward inclination of the camera axis by $30^{\circ}$ and $20^{\circ}$, respectively, has been successfully adopted in a self-calibrating BBA. In the first referenced work it was actually observed that a mix of oblique and nadiral images increases the vertical accuracy of the ground points (although in their experiments resulted in a worse horizontal accuracy), but their results with a pure off-nadir image block without GCP provided similar results with much better XY RMSE. It may be provisionally concluded, as observed also in the work by (James at al., 2020), that mild oblique imaging $\left(15^{\circ}\right)$, without nadir supporting images, is not enough to prevent the dome effect, and that a combination of nadiral and inclined imaging seems better than a single imaging type (either nadiral or inclined). From an operational point of view, this strategy seems also more compelling, the nadiral image block providing a more uniform image scale factor all over the investigated area and reducing occlusions if tall objects are present in the scene. The off-nadir block, on the other hand, provides geometric robustness and increases the accuracy of the on-the-job calibration, preventing or limiting the insurgence of parameter correlation.

The best configuration, under any combination of side-lap and ground control, is LN-CI. Interestingly, this applies to both the GCP and the GNSS case. Moreover, in the best case (LNCI_80\% with 5 and 1 GCP, respectively) GNSS and GCP are equally accurate (1.07 GSD cm against $1.14 \mathrm{GSD} \mathrm{cm}$, respectively).

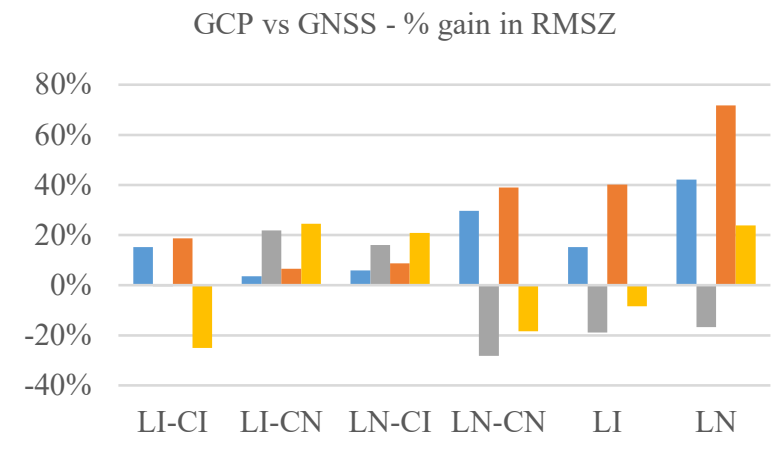

ロ $80 \% 5 / 1 \mathrm{GCP} \square 80 \% 4 / 0 \mathrm{GCP} \square 60 \% 5 / 1 \mathrm{GCP} \square 60 \% 4 / 0 \mathrm{GCP}$

Figure 5. GCP vs GNSS RMSZ gain for each of the six basic configurations and side-lap percentage. Positive values mean

GNSS is more accurate, negative ones the contrary.

Figure 5 presents a more in-depth comparison of the effectiveness of GNSS with respect to GCP in preventing the dome effect i.e., the elevation errors. To this aim, the gap between the two average expected errors is presented as percentage of the GCP case:

$$
\frac{R M S Z_{G C P}-R M S Z_{G N S S}}{R M S Z_{G C P}}
$$

If the GNSS is more effective, the percentage has a positive sign while it has a negative one when the GCP case is more accurate. As can be seen, the GNSS usually provides better performances. In some cases, for the two most rigid configurations (LN-CI and LI-CN) it is just slightly better (4-8\%). In other, for instance LN with $60 \%$ side-lap, is up to $70 \%$ better. The largest gains are in the LN case (top) and in LI and LN-CN. The only cases where GNSS controlled blocks provide worse results are the ones without GCP and without mixed longitudinal and cross strips, as observed before.

It is worth noting that the RMSE results presented so far represent the average entity of the whole "dome effect". In other words, if multiplied by the total area of the survey, it can highlight the total dome (or bowl) volume. It would be interesting to investigate if different configurations produce different dome shapes, in particular with higher or lower maximum errors.

Figure 6 shows the average maximum $Z$ error (maxZE) on check points, expressed in GSD units, for the six configurations with different side-lap and block control separately for the GCP and GNSS case, respectively.

$$
\text { GCP - max Z error (GSD) }
$$

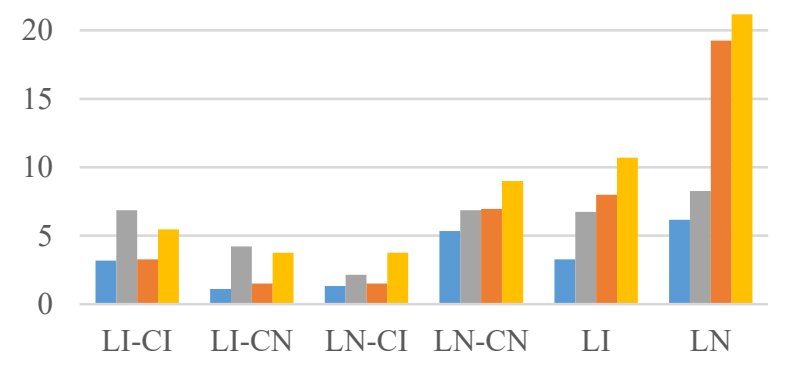

GNSS - max Z error (GSD)

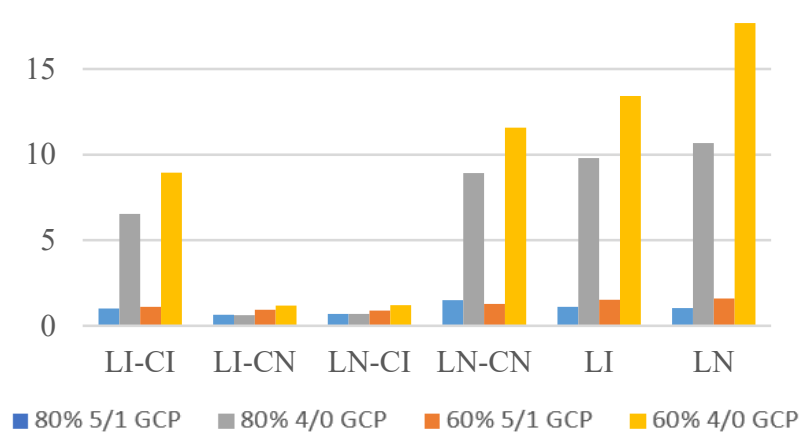

Figure 6. Maximum $Z$ error in GSD units for the six basic configurations in the GCP (top) and GNSS (bottom) cases.

From Figure 6 it is apparent that GNSS is much more effective than GCP to prevent large errors. Indeed, though the worst error case (LN with $60 \%$ side-lap and 4 or no GCP) is the same for both cases and the error size almost identical, on average the advantage of the GNSS is apparent, all the more when one GCP is fixed. In fact, the average value of maxZE is 2 GSD for the GNSS against 7.8 GSD for the GCP case. This is a much larger gap than between the averages for the RMSZ (1.33 against 2.00). The weak point for the GNSS is just the need for at least one GCP, as otherwise the errors are larger than with the corresponding configuration with GCP control (on average, 13.5 GSD for GNSS against 11.4 GSD for GCP). 
Mixed nadiral and inclined configurations (LI-CN and LN-CI) perform best, at the same level in GNSS, slightly better for LN$\mathrm{CI}$ in GCP case. All nadiral LN-CN and all-inclined LI-CI are not much better than a single LI configuration.

Figure 7 refers to a comparison between GNSS and GCP effectiveness in reducing the max errors, in a way similar to Figure 5. This time, however, the gap between the two expected $\max \mathrm{Z}$ errors is presented, as percentage of the max $\mathrm{Z}$ error in the GCP case. As before, if the GNSS is more effective in keeping the error bounded, the percentage has a positive sign while is negative in case the GCP case is more accurate.

\section{GCP vs GNSS - \% gain in max Z error}

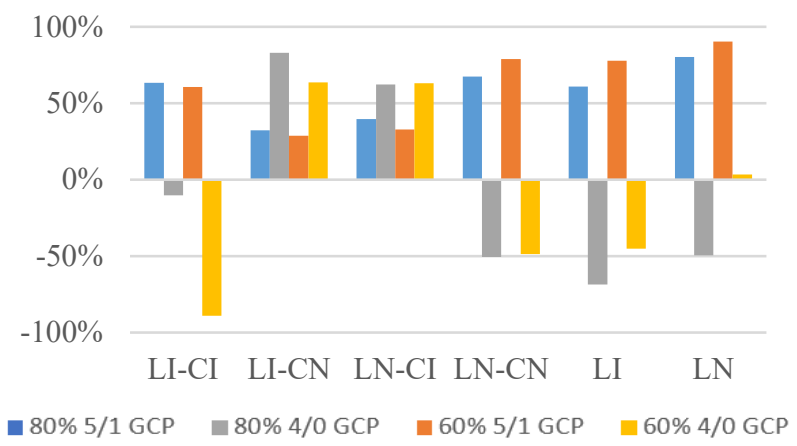

Figure 7. GCP vs GNSS max Z error gain for each of the six basic configurations and side-lap percentage. Positive values imply GNSS smaller errors, negative ones the contrary.

The overall picture conveyed by Figure 7 is similar to Figure 5, as again the GNSS is better in all cases except with LI-CI and LN-CN, LI and LN when no GCP is available. The main difference is the size of the gaps, that are almost all quite significant, mostly between $60 \%$ and $90 \%$ with one GCP at block centre. The only two partial exceptions (LN-CI and LI-CN at $60 \%$ side-lap) show an increase limited to around $30 \%$. Still, even without the GCP at block centre, when the configuration is strong enough to allow unbiased estimation of the principal distance, the gain is quite large (83\% in LI-CN configuration and $62 \%$ in LNCI).

\subsection{Effect of block shape: BWH_1 vs BWH_2}

As illustrated in Section 2, the simulations aimed also to find out whether, still applying the same two variants of block control with both GCP and GNSS, slimming the block from nine to five (with $80 \%$ side-lap) while keeping the longitudinal strip length unchanged, affects the sensitivity to systematic errors in elevation. In principle, one can expect that, the more the block slims out i.e., the more the case looks like corridor mapping, the more deformations in elevations are likely to occur, especially for the GNSS configurations.

Figure 8 presents the gap between the average errors (RMSZ) in BWH_1 and BWH_2 as percentage of the BWH_1 block shape, computed as:

$$
\frac{R M S Z_{B W H \_1}-R M S Z_{B W H_{-} 2}}{R M S Z_{B W H \_1}}
$$

Positive percentages mean the BWH_2 shape is more effective in maintaining limited the RMSZ, negative ones the contrary. The top plot refers to block control with GCP, the bottom one to control with GNSS.

The two plots are quite different. In the GCP case, the changes in RMSZ are almost all insignificant, below $10 \%$. The only exception is the $80 \%$ side-lap case with 4 GCP that, in four cases (LI-CI, LI-CN, LN-CN and LN) sees an RMSZ between $17 \%$ and $19 \%$ better in BWH_2, i.e. in the "slimmer" block. This is broadly in line with expectations as, with 5 GCP, the GCP at the block centre causes a "dampening" of the dome while this is not the case with 4 GCP only. However, the fact that this doesn't happen also in the twin case ( $60 \%$ with 4 GCP) is puzzling. As of today, we could not yet find a convincing explanation for this behaviour.

It is worth noting that, in some cases, the slimmer BWH_2 performs a bit better than the BWH_1 counterpart, although slightly ( $2 \%-3 \%$ except for LI-CN with 4 GCP where the gain is ca. $9 \%$ ). Even if the location of the GCP in the thinner photogrammetric blocks are almost the same along the longitudinal strip direction, being the width of the area surveyed smaller, so is the check points average distance to the nearest GCP. This reduces, in the more rigid blocks, the "dome effect". This behaviour is confirmed in the even slimmer image block (see next section with BWH_3).

\section{GCP : RMSZ change from BWH_1 to BWH_2}

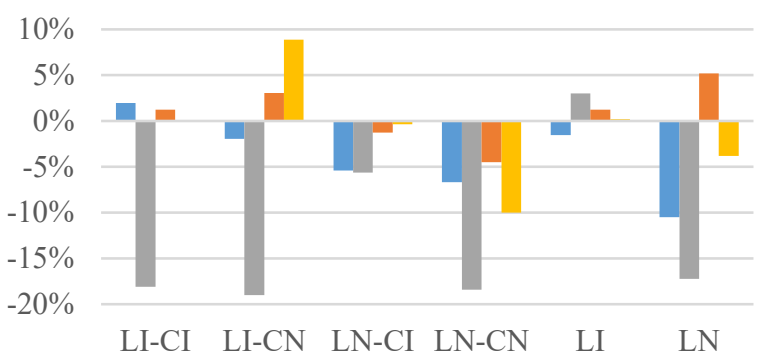

GNSS: RMSZ change from BWH_1 vs BWH_2

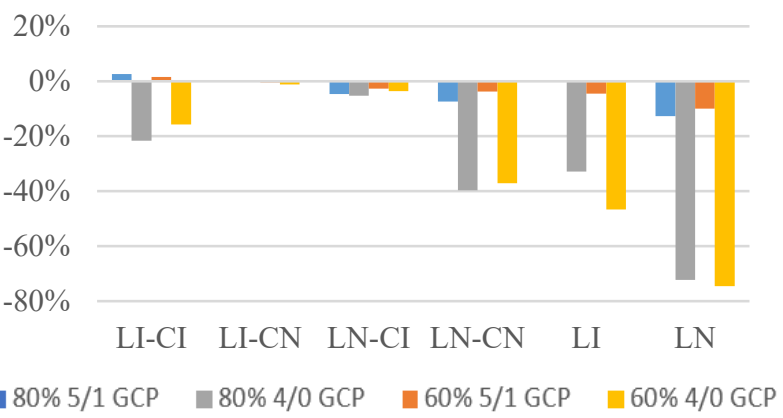

Figure 8. BWH_1 vs BWH_2 RMSZ change for each of the six configurations and side-lap percentage. Top: GCP case; bottom: GNSS case. Positive values correspond to BWH_1 being less accurate, negative ones to the contrary.

In the GNSS case the picture is clearer. For the mixed configurations LI-CN and LN-CI there is practically no drop in accuracy (all changes below 5\%), irrespective of side-lap and of fixing or not a GCP at block centre. Also, when one GCP at block centre is available, the accuracy decrease with a slimmer block is quite limited, with top value of $13 \%$ in the LN configuration. Only if no GCP on ground are available, as it has been found in Figure 2 and 3, for both the $80 \%$ and $60 \%$ side-lap, the accuracy decreases significantly. From around $20 \%$ in LI-CI, it grows to about $40 \%$ in LN-CN and LI and finally tops at $74 \%$ in LN.

\subsection{Effect of block shape: BWH_1 vs BWH_3}

In this Section the results are shown with a larger slimming of the block: from nine longitudinal strips to three only (when $80 \%$ side-lap is adopted, otherwise two strip only). As in Section 3.2, Figure 9 presents the gap between the two average expected 
errors (RMSZ) in BWH_1 and BWH_3 as percentage of the BWH_1 block shape, computed as in eq. (2). As before, negative percentages mean the BWH_1 block shape is more effective in keeping limited the RMSZ compare to BWH 3 shape. The top plot refers to block control with GCP, the bottom one to GNSSassisted control.

GCP : RMSZ change from BWH_1 to BWH_3

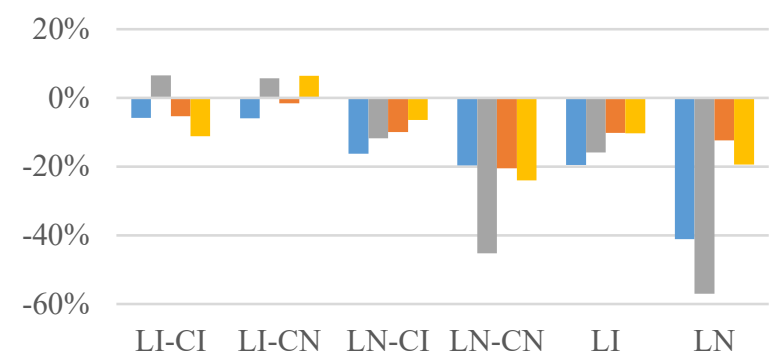

GNSS : RMSZ change from BWH 1 to BWH 3

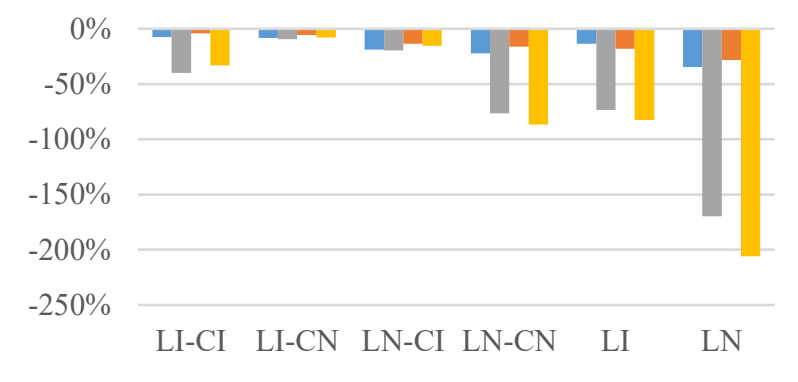

-80\% 5/1 GCP $\square 80 \% 4 / 0 \mathrm{GCP}=60 \% 5 / 1 \mathrm{GCP} \square 60 \% 4 / 0 \mathrm{GCP}$

Figure 9. BWH_1 vs BWH_3 RMSZ change for each of the six configurations and side-lap percentage. Top: GCP case; bottom: GNSS case. Positive values correspond to BWH_1 being less accurate, negative ones to the contrary.

As in 3.2, the two plots are quite different. In the GCP case, three patterns seem to emerge. Changes in RMSZ are almost insignificant, below $10 \%$, for LI-CI and LI-CN configurations. In LN-CI and LI the loss of accuracy grows from weaker to stronger cases, though still not larger than $20 \%$. Finally, in LN-CN and LN the RMSZ loss in BWH_1 is between $45 \%$ and $57 \%$ for $80 \%$ side-lap with $4 \mathrm{GCP}$ and below $20 \%$ in all the other cases bar one. As before, these results are puzzling as the $60 \%$ side-lap with 4 GCP seem unaffected compared to the $80 \%$ twin case. In the LN configuration a large loss is also experienced by the strongest block $(80 \%, 5 \mathrm{GCP})$ while weaker ones are less affected, which is counterintuitive. As of today, we could not yet find a convincing explanation for this behaviour.

In the GNSS case, the pattern is at first glance simply a scaled version of the BWH_1 to BWH_2 of Figure 8 (bottom). While the individual ratios between corresponding configurations are not all the same, broadly speaking a loss factor close to 3 when block shape changes from BWH_2 to BWH_3 is a fair approximation. It is worth noting that, in these cases (the actual scale of the chart in Figure 9 does not allow to notice it) the percentage loss in accuracy with respect to the BWH 1 shape, is also for the "best" LN-CI configuration, quite significant (ca. $20 \%$ ). Much shorter cross strips seem to provide less effective constraints in limiting the "dome effect" and are less efficient in reducing the principal distance correlation with the other parameters. For very thin and long block shapes (e.g., in corridor mapping), increasing side-lap and using off-nadir images improves the GNSS case: the accuracy deterioration with respect to the BWH_1 image block is ca. $14 \%$ vs. the corresponding
(5GCP) in the GCP controlled block, where the RMSZ increase is ca. $20 \%$.

For all the other configurations (e.g., LI with $60 \%$ side-lap) the GCP control case seems a better choice (the RMSZ increase is in this case ca. $10 \%$ vs the GNSS case $18 \%$ growth).

\section{CONCLUSIONS}

The so called "dome" (or "bowl") effect is a systematic photogrammetric block deformation due to inaccurate estimation of the camera parameters. Many researchers, in the recent past, investigated this phenomenon, especially as far as block design is concerned. To avoid projective coupling within E.O. and I.O. parameters, pre-calibration would be advisable, but do not always seems practicable, for instance in UAV surveys where on-the-job calibration is usually preferred.

One of the paper goals was to find out benefits and possible limits, in this context, of oblique imaging. It turns out, in this respect, that control by GCP and GNSS behave differently. In GCP case, both a larger side-lap and the additional GCP at block centre significantly improve the accuracy, especially for weaker block configurations: up to $+290 \%$ for $\mathrm{LN}$ configurations, a standard nadiral longitudinal block without cross strips. A combination of nadiral and inclined imaging seems better than a single imaging type (either nadiral or inclined). Indeed, the best performing configurations, according to the presented results, were LN-CI and LI-CN. Cross strips proved effective even if flown with low overlaps ( $50 \%-50 \%$ in our tests).

In the GNSS case, the best performing configurations are still LN-CI and LI-CN. However, in the four remaining configurations, there is a large gap between cases where at least one GCP is fixed or not. With this single GCP the accuracy is generally significantly better (from $15 \%$ to $40 \%$ ) than the corresponding configuration in GCP. Without it, the GCP case might be up to $30 \%$ better.

It can be speculated that mild oblique imaging $\left(15^{\circ}\right)$ in itself is not enough to prevent the dome effect and must be complemented by cross strips. In fact, other authors (e.g., Taddia et al., 2020; Stott et al., 2020) reported no effect using more inclined $\left(20^{\circ}\right.$ $30^{\circ}$ ) images without any GCP. Additional investigations will be performed in the future to assess whether there is a minimum inclination angle that might ensure that, also in GNSS case without GCP, systematic errors in elevation can be avoided. A second point that emerges is that, when relying on GNSSdetermined camera stations for block control, the adopted block geometry must guarantee successful determination of camera parameters. In our test, this requirement is satisfied by either LNCI or LI-CN configurations. Otherwise, it is highly probable that elevations will be biased, unless at least one GCP is fixed: this is in any case a worthy check and a safeguard that, whenever feasible, should be adopted.

From an operational point of view, this strategy seems also more compelling, the nadiral image block providing a more uniform image scale factor all over the investigated area and reducing occlusions if tall objects are present in the scene. The off-nadir block, on the other hand, provides geometric robustness and increase the accuracy of the on-the-job calibration preventing or limiting the insurgence of parameter correlation. As far as the current simulations results are concerned, it should be noted that, in this configuration, at least for the BWH_1 block shape, using lower longitudinal strip side-lap (i.e., $60 \%)^{-}$and with cross strips flown with very low overlaps $(50 \%-50 \%)$, still provides very good results. In other words, even with a limited total amount of images, this specific image block configuration is very efficient in limiting the systematic "dome effect". 
As far as block shape is concerned, a slimmer block is more subject to deformations in the GNSS case than with the GCP case. Moreover, as pointed out in the previous sections, in these cases providing cross strips throughout the area to be surveyed is, at the very least, impractical, being only longitudinal strips the most natural choice. The best choice according to our experiments, as far as the application approaches a corridor mapping scenario, and GNSS-assisted triangulation is required (e.g., because of hardly accessible areas for GCP survey), is therefore to acquire the images considering an off-nadir (inclined pose) geometry, increasing the number of longitudinal strips (i.e., increasing their side-lap) and possibly providing at least one GCP.

\section{REFERENCES}

Agisoft (2021). Metashape Official Webpage https://www.agisoft.com/ (last accessed 02.04.2021).

Carbonneau PE, Dietrich JT. 2017. Cost-effective non-metric photogrammetry from consumer-grade UAS: implications for direct georeferencing of structure from motion photogrammetry. Earth Surface Processes and Landforms 42: 473-486

Cramer, M., Stallmann, D., Haala, N., 2000. Direct georeferencing using GPS/inertial exterior orientations for photogrammetric applications. Int. Arch. Photogramm. Remote Sens., XXXIII-B3/1, 198-205.

Cramer, M., Przybilla, H. J., Zurhorst, A., 2017. UAV cameras: Overview and geometric calibration benchmark. Int. Arch. Photogramm. Remote Sens. Spatial Inf. Sci., 42, 85.

Daakir, M., Pierrot-Deseilligny, M., Bosser, P., Pichard, F., \& Thom, C. (2015). UAV onboard photogrammetry and GPS positioning for earthworks. Int. Arch. Photogramm. Remote Sens. Spatial Inf. Sci., 40(3), 293.

Fraser, C., 2018. Camera calibration considerations for UAV photogrammetry. Presented at the ISPRS TC II Symposium 2018, Riva del Garda, Italy.

Forlani, G., Diotri, F., di Cella, U. M., Roncella, R., 2020. UAV Block Georeferencing and Control by On-Board GNSS Data. Int. Arch. Photogramm. Remote Sens. Spatial Inf. Sci., 43, 9-16.

Forlani, G., Diotri, F., Cella, U.M. di, Roncella, R., 2019. Indirect UAV Strip Georeferencing by On-Board GNSS Data under Poor Satellite Coverage. Remote Sens. 11, 1765

Girod, L. M. R., Filhol, S. V. P., 2020. Absolute Coregistration and Doming Correction in Adverse Conditions, or how to Retrieve Snow Depth from Drone Flights. ISPRS Ann. Photogramm. Remote Sens. Spatial Inf. Sci., 3, 375-379.

James, M.R., Robson, S., 2014. Mitigating systematic error in topographic models derived from UAV and ground-based image networks. Earth Surf. Process. Landf. 39, 1413-1420.

James, M. R., Robson, S., Smith, M. W., 2017. 3-D uncertaintybased topographic change detection with structure-from-motion photogrammetry: precision maps for ground control and directly georeferenced surveys. Earth Surface Processes and Landforms, 42(12), 1769-1788.

James, M. R., Antoniazza, G., Robson, S., \& Lane, S. N. (2020). Mitigating systematic error in topographic models for geomorphic change detection: accuracy, precision and considerations beyond off-nadir imagery. Earth Surface Processes and Landforms, 45(10), 2251-2271.

Radford, C.R., Bevan, G., 2019. A CALIBRATION WORKFLOW FOR" PROSUMER" UAV CAMERAS. Int. Arch. Photogramm. Remote Sens. Spatial Inf. Sci., XLII-2/W13, 553-558.

Sanz-Ablanedo, E., Chandler, J.H., Rodríguez-Pérez, J.R., Ordóñez, C., 2018. Accuracy of Unmanned Aerial Vehicle (UAV) and SfM Photogrammetry Survey as a Function of the Number and Location of Ground Control Points Used. Remote Sens. 10, 1606

Sanz-Ablanedo, E., Chandler, J.H., Ballesteros-Pérez, P., Rodríguez-Pérez, J.R., 2020. Reducing systematic dome errors in digital elevation models through better UAV flight design. Earth Surface Processes and Landforms. 45(9), 2134-2147.

Taddia, Y., Stecchi, F., Pellegrinelli, A., 2020. Coastal mapping using Dji Phantom 4 RTK in post-processing kinematic mode. Drones, $4,9$.

Wackrow, R., Chandler, J.H., 2008. A convergent image configuration for DEM extraction that minimises the systematic effects caused by an inaccurate lens model. Photogramm. Rec. 23 (121), 6-18.

Nesbit, P.R.; Hugenholtz, C.H. Enhancing UAV-SfM 3D Model Accuracy in High-Relief Landscapes by Incorporating Oblique Images. Remote Sens. 2019, 11, 239.

Harwin, S., Lucieer, A., Osborn, J., 2015 The Impact of the Calibration Method on the Accuracy of Point Clouds Derived Using Unmanned Aerial Vehicle Multi-View Stereopsis. Remote Sens., 7, 11933-11953.

Stott E., Williams R. D., Hoey, T. B., 2020. Ground Control Point Distribution for Accurate Kilometre-Scale Topographic Mapping Using an RTK-GNSS Unmanned Aerial Vehicle and SfM Photogrammetry, Drones, 4, 3, (55).

Tournadre, V., Pierrot-Deseilligny, M., Faure, P. H., 2015. UAV linear photogrammetry. Int. Arch. Photogramm. Remote Sens. Spatial Inf. Sci, 40(3), 327. 